\title{
A Glutamate Receptor-Interacting Protein homolog organizes muscle guidance in Drosophila
}

\author{
Laura E. Swan, ${ }^{1,5}$ Carolin Wichmann, ${ }^{1,5}$ Ulrike Prange, ${ }^{1}$ Andreas Schmid, ${ }^{1}$ Manuela Schmidt, ${ }^{1}$ \\ Tobias Schwarz, ${ }^{2}$ Evgeni Ponimaskin, ${ }^{2}$ Frank Madeo, ${ }^{3}$ Gerd Vorbrüggen, ${ }^{4}$ and Stephan J. Sigrist ${ }^{1,6}$ \\ ${ }^{1}$ European Neuroscience Institute Göttingen, Max-Planck-Society, 37073 Göttingen, Germany; ${ }^{2}$ Department of Neuro and \\ Sensory Physiology, Medical School, University of Göttingen, 37073 Göttingen, Germany; ${ }^{3}$ Institute for Physiological \\ Chemistry, University of Tübingen, 72076 Tübingen, Germany; ${ }^{4}$ Max-Planck-Institute for Biophysical Chemistry, \\ 37077 Göttingen, Germany
}

During Drosophila embryogenesis, developing muscles extend growth-cone-like structures to navigate toward specific epidermal attachment sites. Here, we show that the homolog of Glutamate Receptor-Interacting Proteins (DGrip) acts as a key component of proper muscle guidance. Mutations in dgrip impair patterning of ventral longitudinal muscles (VLMs), whereas lateral transverse muscles (LTMs) that attach to intrasegmental attachment sites develop normally. Myoblast fusion, stabilization of muscle contacts, and general muscle function are not impaired in the absence of DGrip. Instead, the proper formation of cellular extensions during guidance fails in dgrip mutant VLMs. DGrip protein concentrates at the ends of VLMs while these muscles guide toward segment border attachment sites. Conversely, LTMs overexpressing DGrip form ectopic cellular extensions that can cause attachment of these muscles to other muscles at segment borders. Our data suggest that DGrip participates in the reception of an attractive signal that emanates from the epidermal attachment sites to direct the motility of developing muscles. This dgrip phenotype should be valuable to study mechanistic principles of Grip function.

[Keywords: dgrip; muscle; muscle guidance; Glutamate Receptor-Interacting Protein; Drosophila]

Received October 4, 2003; revised version accepted November 26, 2003.

The establishment of specialized cell-cell junctions plays a determining role in the formation of mature patterned organs in all multicellular organisms. The most prominent examples are synaptic connections, which are formed either between neurons or between neurons and other cells, for example, muscles. Cells form extensions such as growth cones, lamellipodia, or filopodia, which they use to sense specific guidance cues and to finally anchor at the relevant target cells. In Drosophila, developing muscles grow growth-cone-like projections to navigate toward specific epidermal attachment sites (Baylies et al. 1998; Volk 1999). Drosophila muscles are grouped into two categories. One muscle type, which includes the lateral transverse muscles (LTMs), is characterized by single muscle fibers attaching to a single epidermal tendon cell. The other type, indirectly attaching muscles, including the ventral longitudinal muscles (VLMs), converges with several muscle fibers on single tendon cell, recruiting extracellular matrix, to which

\footnotetext{
${ }^{5}$ These authors contributed equally to this work.

${ }^{6}$ Corresponding author.

E-MAIL ssigris@gwdg.de; FAX 49-551-3912346.

Article published online ahead of print. Article and publication date are at http://www.genesdev.org/cgi/doi/10.1101/gad.287604.
}

they adhere (Prokop et al. 1998). Recent evidence showed that these tendon cells, also called apodemes, are the source of secreted Slit protein. Slit is sensed as a positive guidance cue by Robo receptors expressed in the nascent VLMs. Furthermore, experimentally induced overexpression of Robo receptors causes LTMs to extend toward Slit expressing tendon sites (Kramer et al. 2001). In addition to the Robo/Slit-system controlling VLM guidance, the Derailed receptor tyrosine kinase controls LTM guidance (Callahan et al. 1996). Interestingly, both systems also have firmly established roles in axonal guidance processes, suggesting a common mechanistic basis for cellular motility of muscles and neurons.

Guidance processes are controlled by a diverse array of signaling proteins, with spatiotemporal activity that is subject to subtle regulation (Dickson 2002; Huber et al. 2003). How the cellular metabolism of such supramolecular signaling complexes is organized is subject of intense investigation. Proteins containing PDZ domains, a protein-protein interaction domain of $\sim 90$ amino acids (Willott et al. 1993; Jesaitis and Goodenough 1994), recruit components of a signaling network into larger molecular complexes in order to allow rapid and specific intracellular signaling (Bilder 2001; Sheng and Sala 2001). GRIP family proteins (GRIP1 and ABP/GRIP2) 
contain six or seven PDZ domains in tandem. They were first identified via an interaction of their fifth PDZ domain with the C-terminal sequence (ESVKI) of the GluR2 AMPA receptor subunit (Dong et al. 1997; Srivastava et al. 1998; Wyszynski et al. 1999) and are suggested to participate in the synaptic localization of AMPA receptors. Interfering with the interaction between GRIPs and GluR2/3 prevents AMPA receptor recruitment to the synapse in vitro (Dong et al. 1997; Osten et al. 2000; Xia et al. 2000). GRIP has also been identified as a binding partner of both ephrin receptors and ligands (Torres et al. 1998; Brückner et al. 1999; Lin et al. 1999; Contractor et al. 2002), ARF-GAP GIT1 (Ko et al. 2003), the kinesin motor protein KIF1A, and liprin- $\alpha$ (Ko et al. 2003; Wyszynski et al. 2002). Despite this information, the cell biological basis of GRIP function is only poorly understood. Biochemically, GRIP1 is slightly enriched in synaptic preparations but also is strongly expressed in intracellular compartments, including putative transport vesicles for glutamate receptors (Wyszynski et al. 1998, 2002; Dong et al. 1999a). GRIPs have been suggested to mediate (1) the transport of glutamate receptors directly (Dong et al. 1997; Wyszynski et al. 2002), (2) the stabilization of receptors within postsynaptic densities (Osten et al. 2000), or (3) the stabilization of intracellular stores and/or participation in sorting decisions for the destruction or recycling of internalized receptors (Shi et al. 2001; Hirbec et al. 2003). Genetic analysis in mice has shown that GRIP1 function is already required early during development, because a GRIP1 knockout was embryonic lethal at day 12 and the embryos suffered from defects in junction formation between dermis and epidermis (Bladt et al. 2002).

In the present study, we present evidence that CG14447, the single GRIP homolog in Drosophila and therefore named DGrip, participates in muscle development during embryogenesis. Loss of dgrip function caused severe defects in VLM but not LTM patterning. DGrip is required for the guidance of developing VLMs toward the apodemes. Other processes such as myoblast fusion, stabilization of muscle attachments, and muscle function per se are not affected in dgrip mutant embryos and larvae. Mesodermal expression of DGrip using transgenes rescued the dgrip mutant phenotype. Consistent with its specific function in VLM guidance, DGrip protein progressively concentrates at the ends of these muscles as they establish contact to their target position. Furthermore, when DGrip was overexpressed within embryonic mesoderm, LTMs were guided toward ectopic attachment sites at segment borders. The DGrip protein therefore appears to be used by a subset of muscles to direct their motility, likely by transporting and/or localizing signaling components of a novel pathway.

\section{Results}

Drosophila contains a single GRIP homolog that is specifically expressed in developing muscles

As previously noted (Littleton and Ganetzky 2000), the Drosophila genome encodes a single GRIP homolog
(CG14447). CG14447 is represented by several embryonic cDNA isolates (see Materials and Methods; Rubin et al. 2000), which all predict the same protein sequence. Comparison of this sequence with mouse GRIP1 in respect to both position and sequence of PDZ domains clearly identifies CG14447 as a GRIP family member (Fig. 1).

To examine the expression pattern of dgrip, an in situ hybridization against dgrip was performed on Drosophila embryos (Fig. 1). Until germ band extension, no dgrip expression was detected (Fig. 1A). From stage 10 onward, staining in the posterior half of the segments (Fig. 1C-F, arrowheads) is observed, indicating expression in developing somatic muscles (Baylies et al. 1998). In fact, in stage 16 embryos, a strong dgrip mRNA expression is observed specifically within muscles (Fig. 1G, arrowhead), whereas at this stage neither epidermis (Fig. $1 \mathrm{G}$, arrow) nor central nervous system (CNS; Fig. 1F, arrow) seem to express dgrip mRNA.

\section{DGrip mutants show strong patterning defects of VLMs}

The dgrip locus maps to position 5C10 on chromosome $X$ (Adams et al. 2000). To generate mutations, we made use of the P-element insertion P(GT1)BG01736 (Kimmerly et al. 1996) located $2 \mathrm{~kb}$ downstream of the DGrip stop codon. Upon remobilization of the P-element, the small deletion dgrip ${ }^{\text {ex36 }}$ was recovered, in which the whole transcription unit but no other annotated gene is deleted (Fig. 2A). Alternatively, we started from $\mathrm{P}(\mathrm{KG}) 02862$ (Roseman et al. 1995), which is inserted just upstream of the dgrip transcription start and recovered

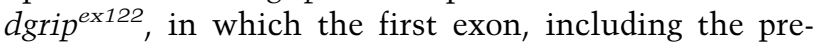
dicted start codon of the dgrip locus, is deleted. Individuals of the genotype $\operatorname{dgrip}^{\text {ex36}} / \mathrm{Y}, \operatorname{dgrip}^{\text {ex122}} / \mathrm{Y}$, and

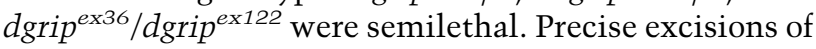
the parental P-lines instead were fully viable and did not present any of the phenotypes observed in $\operatorname{dgrip}^{\text {ex36 }}$ and dgrip ex122 (data not shown). Embryos hemizygous for both dgrip ${ }^{\text {ex36 }}$ and dgrip ${ }^{\text {ex122 }}$ were negative for dgrip mRNA in the in situ hybridization (data not shown). To examine protein expression in dgrip mutant embryos, a polyclonal antibody against PDZ domains 6 and 7 of the protein was affinity-purified (see Materials and Methods). The DGrip encoding cDNAs predict a protein of $112 \mathrm{kD}$. Consistently, Western blot analysis of wild-type Drosophila embryo extracts (stage 10-17) probed with our antibody detected a single band of $\sim 110$ to $120 \mathrm{kD}$ apparent size, which comigrated with recombinant DGrip expressed in insect cells. In contrast, embryo extracts derived from a dgrip ${ }^{\text {ex122 }}$ homozygous strain were negative for DGrip protein on Western blot (Fig. 2B). We therefore conclude that both $d_{g r i p}{ }^{e \times 36}$ and $d_{g r i p}{ }^{\text {ex122 }}$ represent protein null alleles of the dgrip locus. Consistently, both alleles resulted in identical phenotypes (see below). Moreover, phenotypes were identical in dgrip $^{\text {ex122} / Y ~ i r r e s p e c t i v e ~ o f ~ w h e t h e r ~ a n i m a l s ~ w e r e ~ o b-~}$ tained from dgrip ${ }^{\text {ex122 }}$ homozygous or heterozygous mothers. Examining this together with the in situ results 

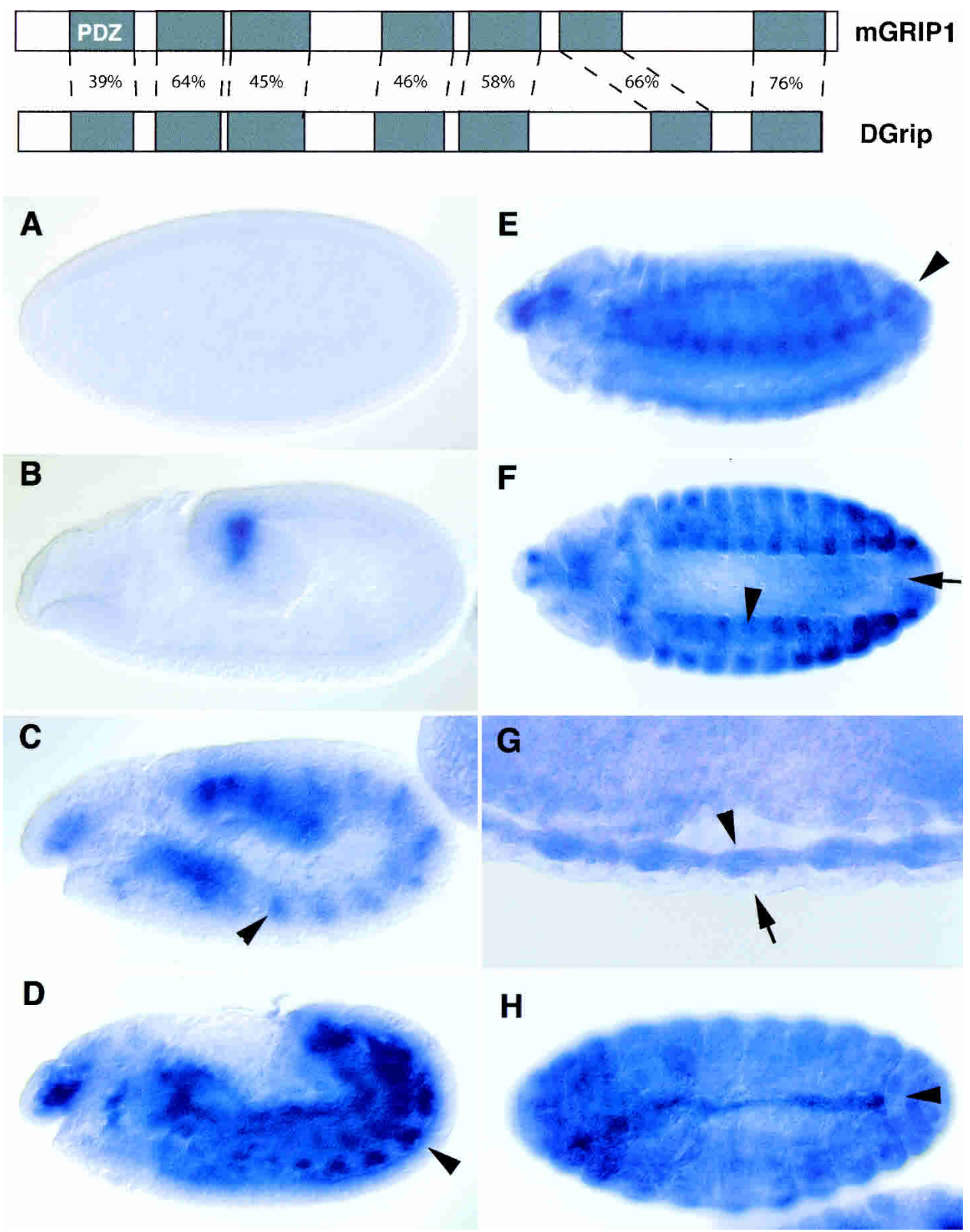

Figure 1. Embryo in situ hybridization for dgrip. (Top) Comparison between mouse GRIP1 and Drosophila protein CG14447 (DGrip), which both encode seven individually conserved PDZ domains. Sequence similarity between corresponding PDZ domains is indicated in percentages. $(A-H)$ In situ hybridization of dgrip. Embryonic stages shown are as follows: $3(A), 6(B), 10(C), 12(D), 13 / 14(E$ and $F$; $F$ shows ventral view), $16(G$; dorsal view of ventral longitudinal muscles [VLMs]), and 17 ( $H$; dorsal view). The dgrip mRNA accumulates in the precursors of the VLMs $(C-F$, arrowheads), resulting in a strong expression within VLMs after the formation of attachments $(G$, arrowhead). Epidermis $(G$, arrow $)$ and CNS $(F$, arrow $)$ do not show detectable dgrip expression. At stage $17(H)$, cardioblasts of the dorsal vessel show strong expression of dgrip mRNA (arrowhead) and of DGrip protein (data not shown). dgrip ${ }^{\text {ex36/Y }}$ embryos did not express any detectable dgrip mRNA (data not shown).

(Fig. 1), we therefore conclude that no maternal activity of DGrip is present and that both alleles thus establish true DGrip null situations in embryos.

To examine the embryonic development of mesoderm in the absence of DGrip activity, the somatic muscle pattern of mutant embryos was visualized by myosin stainings. Control embryos showed the typical pattern of somatic muscles (Fig. 2C, WT). However, an abnormal patterning of the VLMs was easily detected in embryos of the genotype dgrip ${ }^{\text {ex36 }} / \mathrm{Y}$, dgrip ${ }^{\text {ex122 }} / \mathrm{Y}$ (Fig. 2C, asterisks), and dgrip ${ }^{e \times 36} /$ dgrip $^{\text {ex122 }}$ (data not shown). The defective VLMs of the mutants appeared rounded instead of stretched between the attachment sites at the segment borders. Mesodermal expression of DGrip using the 24Bgal4 (Fig. 3D) or twist-gal4 driver (data not shown) rescued the VLM patterning defect of dgrip ${ }^{\text {ex36 }} / \mathrm{Y}$ (Fig. 3C) 


\section{(A)}

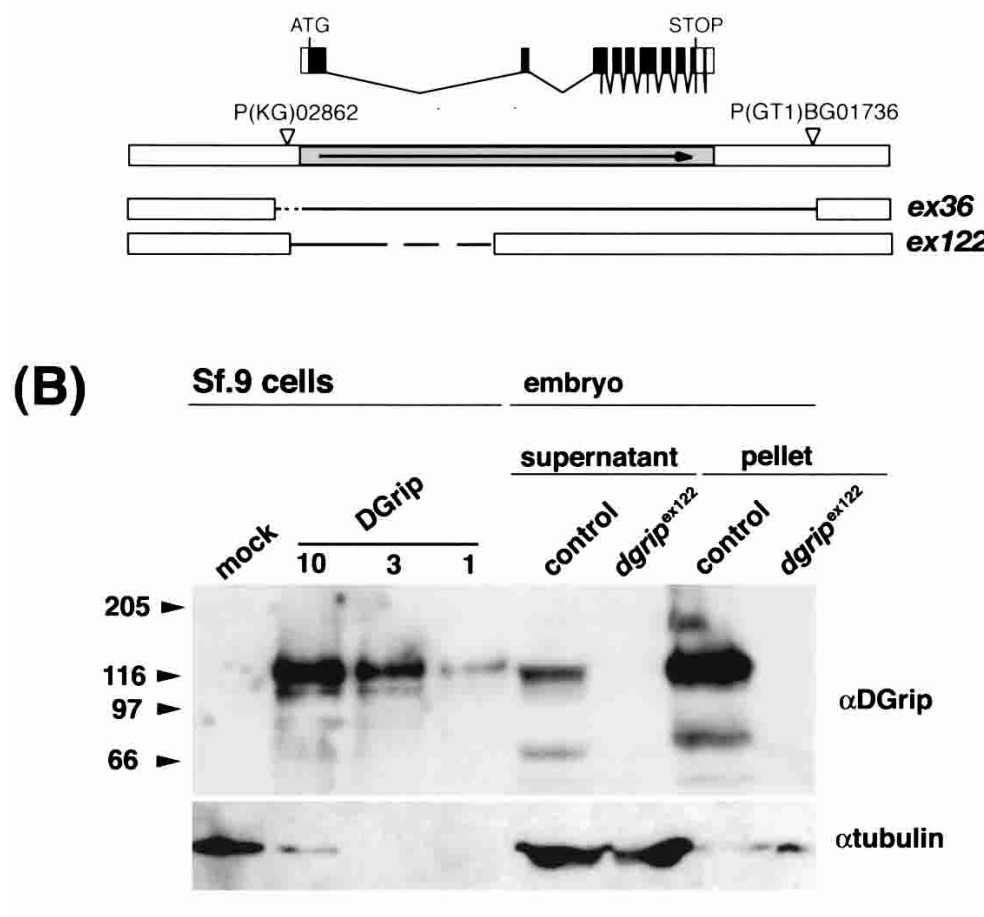

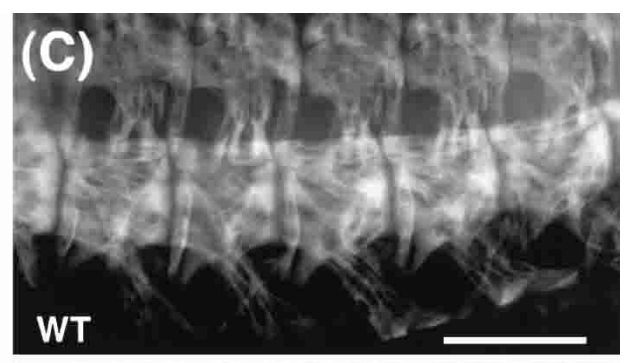
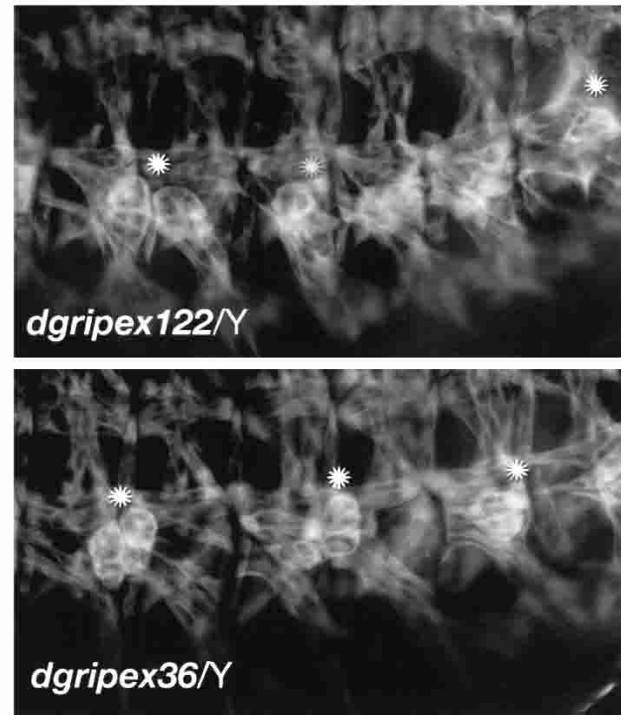

Figure 2. Specific muscle defects in dgrip mutants. (A) Genetic analysis of dgrip. By mobilization of transposon P(GT1)BG01736 2 kb downstream of the DGrip stop, deficiency dgrip ${ }^{\text {ex36 }}$ eliminating the full dgrip ORF was recovered. P(KG)02862, located 500 bp upstream of the DGrip start codon, gave deficiency dgrip ${ }^{\text {ex122 }}$, which deletes the first exon of DGrip, including the putative start codon. Both deficiencies resulted in identical phenotypes, which were rescued by mesodermal expression of DGrip using the $24 B$-gal4 or twist-gal4 driver lines. Precise excisions of the parental P-lines gave wild-type phenotypes. (B) Western blot probed with affinity purified anti-DGrip (top, see Materials and Methods) and anti-Tubulin as a loading control (bottom). Embryos (5 to $15 \mathrm{~h}$ ) were homogenized under low-detergent conditions ("supernatant") and the pellet was solubilized under high detergent ("pellet"); for details, see Materials and Methods. A band of $\sim 120 \mathrm{kD}$ (predicted size for DGrip $112 \mathrm{kD}$ ) is detected in wild-type embryo extracts, whereas no signal is present in identically produced embryonic extracts from dgrip ${ }^{\text {ex } 122}$ homozygous flies, proving this band represents DGrip. Embryonic DGrip comigrated with DGrip recombinantly expressed in Sf.9 cells (mock: untransfected Sf.9 cells; 10, 3, and 1: dilution series from a dgrip transfected Sf.9 cell extract). DGrip was especially enriched in the pellet fraction after high-detergent extraction. (C) Muscle myosin labeling visualizing the somatic muscle pattern in several hemisegments of late stage 16 embryos. Shown are lateral images of whole-mount embryos. Muscle patterning of ventral longitudinal muscles (VLMs) is clearly defective in the two independent alleles, dgrip ${ }^{\text {ex122 }}$ and dgrip ${ }^{\text {ex36. }}$. Asterisks mark groups of misattached VLMs in dgrip mutants. Bar, $60 \mu \mathrm{m}$.

to the wild-type muscle pattern. We similarly tested the epidermal gal4 driver lines, engrailed-gal4 (A. Brand, University of Cambridge, Cambridge, UK) and stripegal4 (G. Morata, University of Madrid, Madrid, Spain), with the latter specifically expressing in tendon cells. Driving dgrip expression with these both lines did not rescue muscle defects of $d_{\text {grip }}{ }^{\text {ex36/Y }} / \mathrm{Y}$ (data not shown). These results indicate that dgrip is required in the developing muscle but not in the epidermis for proper muscle guidance.

Muscle patterning defects in the absence of DGrip were further characterized on the cellular level by using confocal microscopy. Figure 3 shows that in dgrip mutants, the VLMs differed markedly from the elongated cylindrical appearance of wild-type VLMs (Fig. 3A) and consistently failed to attach at both segment borders (Fig. 3B). The mutant VLMs appeared atypically compact and rounded. Such strongly affected VLMs position themselves randomly more at either the anterior or posterior segment end. In weaker cases, while still attached to both segment borders, the mutant VLMs appeared irregularly shaped and did not align in register at the segment borders, a defect not observed for wild-type VLMs. We observed that $>95 \%$ of all VLMs 6/7 were affected in dgrip mutant embryos, with $40 \%$ of the cells of this VLM type showing a full "rounding up" of the muscle cells. VLMs $12 / 13$ were affected to $80 \%$ with $\sim 10 \%$ fully rounded up. Defects within other muscles apart from the VLMs were less obvious in dgrip mutants. With a frequency of $\sim 5 \%$, another type of indirectly attaching muscle, muscle 8, was strongly affected (Fig. 3B, asterisk). Some defects in segment border attachment were also recognized in other muscles: muscle 4 , the ventral oblique muscles 14 and 30, and the dorsal muscles 1 and 

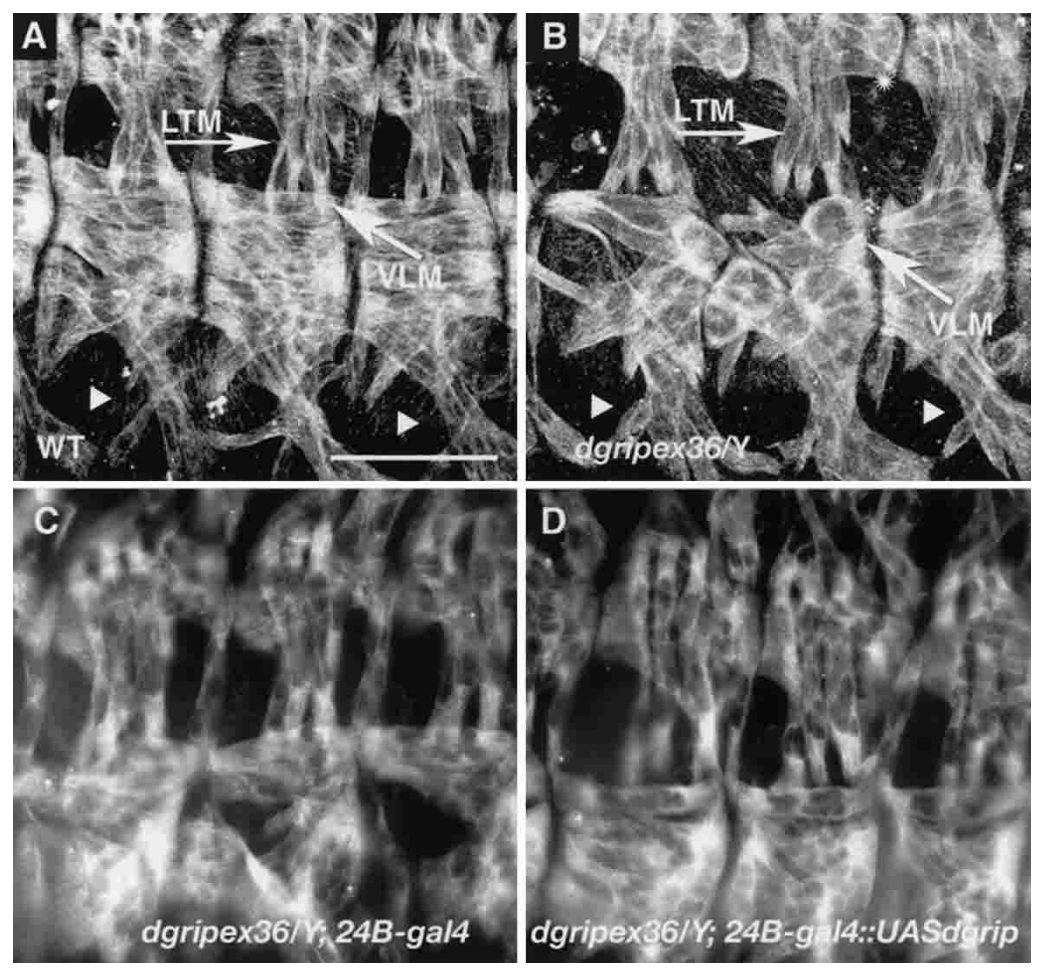

Figure 3. DGrip is essential for patterning ventral longitudinal muscles (VLMs) but not lateral transverse muscles. All images show lateral views on three hemisegments in muscle myosin stainings of late stage 16 embryos. $(A, B)$ Confocal images projected through all muscle layers. Muscles in the VLM area (thick arrows) often round up and no longer attach to both segment borders in dgrip $^{\text {ex36 }} / \mathrm{Y}(B)$. Lateral transverse muscles (thin arrows, cf. $A$ and $B)$ and ventral oblique muscles (arrowheads) are not affected. Occasionally, muscle 8 is also defective (asterisk in $B)$. Bar, $50 \mu \mathrm{m} .(C, D)$ Reexpression of DGrip using a weakly-expressing copy of UAS-dgrip in the dgrip mutant background (D: dgrip ${ }^{\text {ex36}} / \mathrm{Y} ; 24 B$-gal4::UAS-dgrip) rescues the VLM defects obvious in dgrip mutants $(C$ : dgrip $^{e \times 36} / \mathrm{Y} ; 24 B$-gal4).
2 showed milder defects in $\sim 10 \%$ to $20 \%$ of the cells counted. Importantly however, we find directly attaching muscles such as the LTMs to be absolutely unaffected in dgrip mutants (Fig. 3A,B, arrows). In summary, we conclude that DGrip represents an essential component needed to establish the correct patterning within VLMs and to a lesser extent in other indirectly associating muscles during Drosophila embryogenesis.

\section{In the absence of DGrip, muscles differentiate properly and stably attach at ectopic positions}

Defective muscle adhesion, such as that found after interfering with integrin function, often results in muscle detachment. This effect is caused in response to contractile force in the affected muscle, a condition usually fatal in late embryogenesis (MacKrell et al. 1988; Leptin et al. 1989; Brown 1994; Bökel and Brown 2002). However,

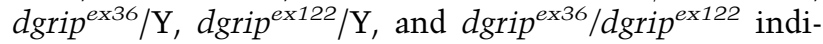
viduals develop into larvae, which maintained the defective VLM pattern observed in embryos (Fig. 4B,C). Within these animals, the embryonically affected muscles had obviously grown and elongated throughout larval development. No sign of muscle degeneration was recognizable. Affected VLMs had produced ectopic intrasegmental attachment sites (Fig. 4B,C, arrowheads) instead of the normal intersegmental attachments in wild type (Fig. 4A, arrowheads). Microscopic inspection of dgrip mutant larvae showed that these attachments form at the inner layer of muscles and not to the epidermis, showing that in the absence of DGrip function, musclemuscle junctions are formed. Such muscle-muscle junc- tions, instead of normal tripartite muscle-muscle-epidermis junctions, have also been reported for other mutants such as kakapo (Prokop et al. 1998). Both in embryos and larvae, even the most strongly affected dgrip mutant VLMs form multiple extensions (Fig. 4D, arrowheads), implying that DGrip-deficient muscles still seek attachments.

That dgrip-deficient larvae were capable of sustained locomotion and the absence of detached muscles strongly suggest that muscle attachments were functional in this mutant. Based on phalloidin stainings (Fig. 4A-C) and electron microscopy (C. Wichmann and S.J. Sigrist, unpubl.), the defective VLMs had normal organization of the contractile apparatus as well. Moreover, escaping dgrip ${ }^{\text {ex36}} / \mathrm{Y}$ dgrip ${ }^{\text {ex122}} / \mathrm{Y}$, or dgrip ${ }^{\text {ex36/ }}$ dgrip $^{\text {ex122 }}$ adults showed a shrunken abdomen (Fig. 4J) and defective head posture, phenotypes likely due to adult muscle patterning defects. However, no other defects-in particular, no signs of a general impairment of cell adhesion-were observed in these animals.

Drosophila muscles are highly differentiated concerning their attachment site and shape (Bate 1990; Jagla et al. 2001). Fate changes among VLM founder cells (Knirr et al. 1999/ could therefore be responsible for the observed muscle phenotype. To examine this possibility, we monitored the VLM pattern in dgrip mutant embryos by staining with the VLM-specific differentiation marker Vestigial (Bate and Rushton 1993). Vestigial was expressed even in the most strongly affected muscles of dgrip $^{\text {ex36}} / \mathrm{Y}$, indicating that the muscles develop according to their proper fate (data not shown). Furthermore, we made use of 5053-gal4, which specifically drives expression in VLM 12 from stage 12 on (Ritzenthaler et al. 
Swan et al.

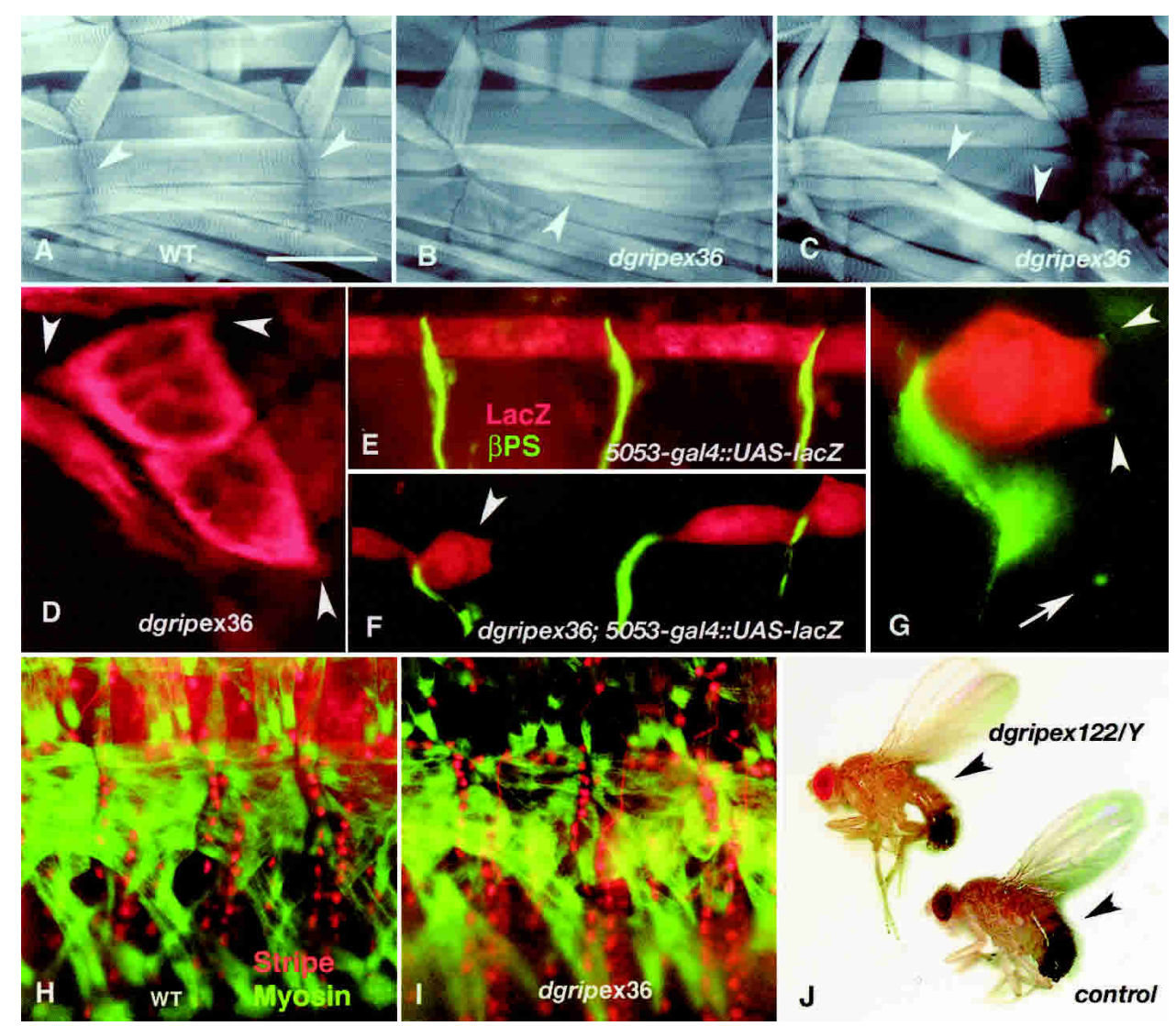

Figure 4. Muscle attachment per se is not affected in dgrip mutants. One hemisegment of third instar larvae in wild type $(A)$ and dgrip $^{\text {ex36 }} / \mathrm{Y}(B, C)$, fillet preparations stained with rhodamine-coupled phalloidin. In dgrip mutants, muscles differentiate a contractile apparatus, and no sign of detachment can be observed. Instead, ectopic muscle-muscle contacts form from misguided ventral longitudinal muscles (VLMs; B, C, arrowheads). Bar, $200 \mu \mathrm{m}$. (D) Confocal image of misguided VLMs in dgrip ${ }^{\text {ex36/Y }}$; arrowheads point to cytoplasmic extensions, indicating the formation of muscle attachments in several directions. (E-G), Stage 17 embryos stained for $\beta$-Gal to show muscle 12 and $\beta P S$-integrin in control $(E)$ and dgrip ${ }^{\text {ex36 }}(F, G)$ background. In dgrip mutants, one muscle 12 has not achieved segment border attachment $(F$, arrowhead). The ectopic muscle contacts from this misguided muscle are integrin positive $(G$, arrowheads; magnification of $F$ ). The lower integrin spot (arrow) in $G$ likely represents an ectopic attachment between unlabeled muscles. $(H-I)$ Stage 17 embryos costained with the apodeme marker Stripe (red) and with myosin (green) in dgrip mutants (I). Apodemes were differentiated as in wild type $(H)$, and muscle pattern was highly impaired $(I)$. (J) Adult dgrip ${ }^{\text {ex122 }}$ male showing a shrunken abdomen (arrowheads) as typical for all dgrip mutant escapers. Bars, $10 \mu \mathrm{m}(D), 30 \mu \mathrm{m}(E)$, and $40 \mu \mathrm{m}(H)$.

2000). Expression of $\beta$-Galactosidase ( $\beta$-Gal) driven by 5053-gal4 develops normally in dgrip mutant embryos (Fig. 4F,G). Collectively, these data show that VLMs still develop proper fate in the absence of dgrip activity. In dgrip mutants, muscle 12 frequently attached to ectopic positions, as shown by $\beta$ PS-integrin staining (Fig. 4E-G, arrowheads in 4G), a marker of muscle attachment sites (Brown 2000). Thus, VLMs lacking DGrip activity are able to attach to ectopic intrasegmental attachment sites by contacting other muscles.

To evaluate myoblast fusion, the number of nuclei was determined in dgrip mutant muscles 12 of stage-17 embryos after Hoechst staining. The number of nuclei was only slightly lower in $d g r i p^{e x 36} / \mathrm{Y}$ than in wild type $(8.7 \pm 1.7$ versus $10.0 \pm 2.0$, respectively; $P<0.08)$. Moreover, because correct muscle attachment is observed in the absence of myoblast fusion (Rushton et al. 1995), a defect in myoblast fusion can be excluded as the primary cause of the muscle defects observed.
Finally, we also examined possible differentiation defects within apodemes. In mutants for the transcription factor stripe, such defects provoke defective attachment of somatic muscles (Frommer et al. 1996; Becker et al. 1997) somewhat similar to those observed in dgrip mutants. However, neither dgrip mRNA (Fig. 1) nor protein (Fig. 6A, arrow in the inset) was detectable in the epidermis throughout the period of attachment formation. Given that DGrip is specifically expressed within the affected muscles and that the defect can be rescued by purely mesodermal expression, a direct role of DGrip in apodeme differentiation appears very unlikely. To positively exclude a role of epidermal cells with respect to the observed phenotype, we stained for the apodeme differentiation markers Delilah (data not shown) and Stripe (Fig. 4H,I) in dgrip mutant embryos of stage 17. Both the number and position of apodemes are unchanged in dgrip mutants (Fig. 4I) compared with wild type (Fig. 4H). Consistent with the formation of pure muscle-muscle junc- 
tions by displaced VLMs, no sign of additional apodemes attached to VLMs was observed in dgrip mutants.

In summary, these results establish that muscle cell differentiation, myoblast fusion, and epidermal attachment sites are not affected by the lack of dgrip activity, leaving the option that the mutant muscles are misguided and fail to properly reach their target sites.

\section{Defective guidance behavior of dgrip mutant muscles}

To explore the possibility that DGrip may function in the guidance of developing VLMs toward their individual epidermal attachment sites, we followed the development of individual VLMs in dgrip mutants. For this task we labeled VLM 12 by expressing UAS-lacZ with the aid of the 5053-gal4 driver (Ritzenthaler et al. 2000).

The developing muscle 12 precursor was first observed at stage 12 in both control and dgrip mutant embryos. Throughout further development, muscles then expand by the integration of naïve myoblasts (Rushton et al. 1995). In control embryos of late stage 13, the growing muscle is still found in the posterior portion of the hemisegments, extending a single cellular extension in- variably toward anterior (Fig. 5A-C). Already at stage 13, VLMs are apparently defective in the absence of DGrip (Fig. 5D-F). Specifically in dgrip mutants, we observed cellular extensions that were pointing in "wrong directions" (Fig. 5F). Moreover, muscle 12 precursor cells in dgrip mutant embryos often appeared bipolar, forming extensions in both anterior and posterior direction (Fig. $5 \mathrm{D}, \mathrm{E})$. In wild type, extensions extend further in stage 14 until they finally contact the epidermal attachment sites at the anterior border of the segment at late stage 14/ early stage 15 (Fig. 5G,H). In dgrip mutants of this stage (Fig. 5I,J), cell extensions often appear collapsed, or if developed, they miss their proper target sites at the segment borders (Fig. 5J). This observation is consistent with the fact that muscles finally are often unable to form proper contact with its normal attachment site. Our results thus explain the appearance of the misattached rounded muscles that are observed in the dgrip mutants from stage 16 onward (cf. Fig. 5M,N, and Figs. 2C, 3B). As mentioned before, in dgrip mutant embryos the patterning of VLM 12 is somewhat less severely affected than that of VLMs 6/7. There was no muscle 6/7-specific driver available to explore the possibility of very likely even more penetrant guidance defects of this muscle pair

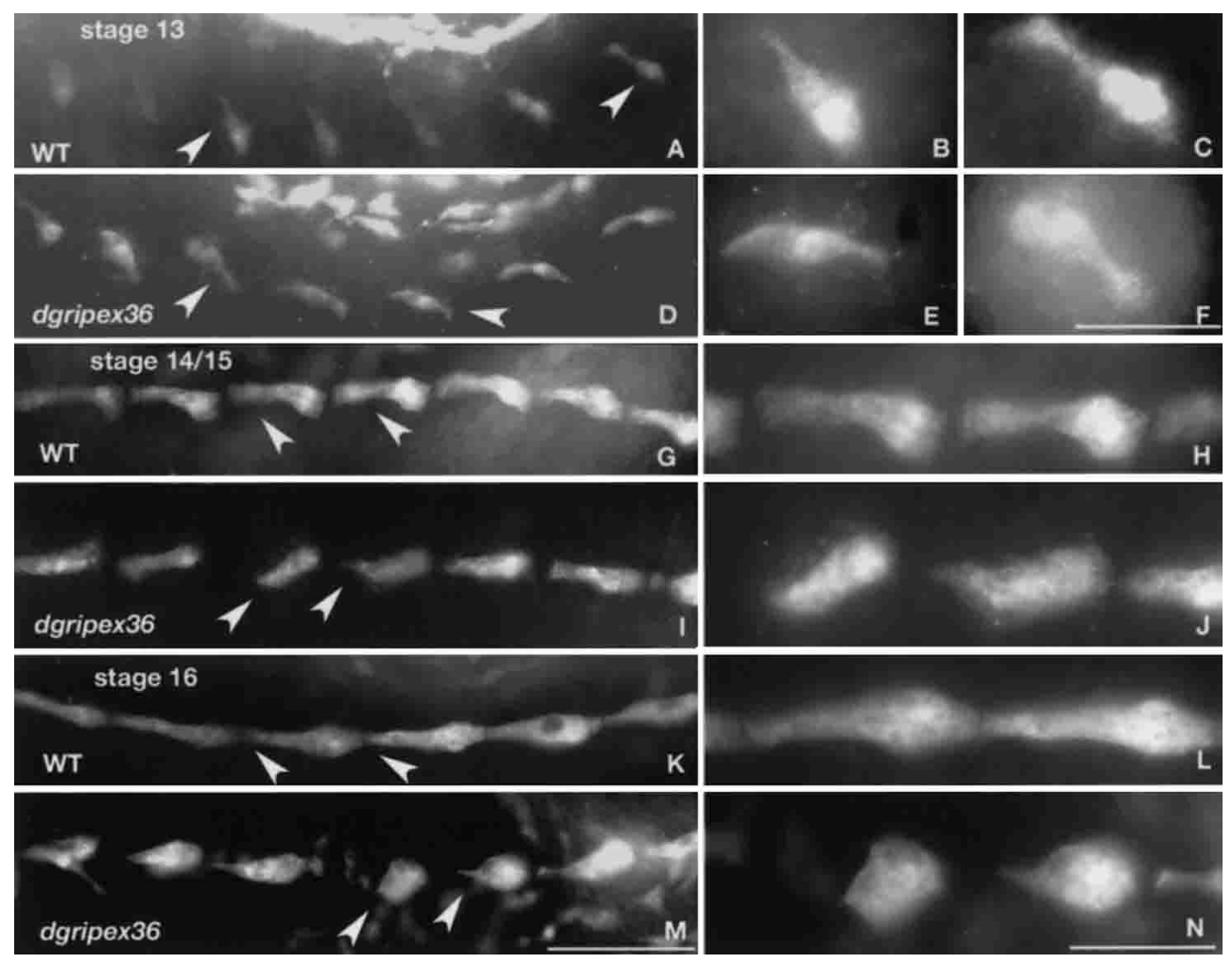

Figure 5. Defective guidance in ventral longitudinal muscles (VLMs) of dgrip mutant embryos. Segment border guidance of muscle 12 , which is visualized in Drosophila embryogenesis by using 5053-gal4 to express $\beta$-Gal specifically in this somatic muscle. Outof-focus staining derives from expression within visceral muscles. Shown are pictures from embryos in stage $13(A-F)$, late stage 14/early $15(G-J)$, and stage $16(K-N)$; right panels show magnifications of individual muscles. In control embryos, developing muscles extend projections anteriorly starting in stage $13(A$, arrowheads, $B, C)$ which broaden and establish first proper contact to the anterior segment border in stage $14 / 15(G$, arrowheads, $H)$. In stage $16(K, L)$, VLM 12 is fully attached. In dgrip mutant embryos, from stage 13 extensions are unusual in shape and often project in wrong directions $(D-F$, arrowheads). Consequently, many dgrip mutant muscles fail to establish proper contact $(I, I ; M, N)$. Bars, $20 \mu \mathrm{m}(F), 60 \mu \mathrm{m}(M)$, and $25 \mu \mathrm{m}(N)$. 
in the dgrip mutant. We conclude that the cellular behavior of dgrip mutant muscles becomes aberrant significantly before the developmental time point at which segment border attachment normally is established. In dgrip mutants, directionality of the cellular extensions normally mediating guidance seems essentially randomized. We conclude this as cells extending in anterior or posterior direction as well as "bipolar" cells are observed in similar quantities. Consistently, dgrip mutant VLMs are found attached to either the anterior or posterior segment border only (Figs. 2C, 3B; for an example of an anterior attachment, see Fig. 4F). We also observed that VLMs in dgrip mutants often stretch over segment borders (data not shown), indicating that the muscles have missed their attachment sites at the segment borders. These results are consistent with our interpretation that prior to any attachment, during stage 13, dgrip mutant muscles fail to respond to attractive cues by directing the outgrowth of cellular extensions (Fig. 5D). Our results thus suggest a direct role of DGrip to mediate the response to an essential attractive signal within the developing muscles. This attractive signal seems to emanate from the segment border in order to direct and/or stabilize cellular extensions of developing muscles.

\section{DGrip accumulates in discrete compartments at muscle ends}

To correlate the embryonic expression with the mutant phenotype, we stained embryos for DGrip protein expression by using our anti-DGrip antibody. In immunostainings of both dgrip ex36 $\mathrm{Y}$ and dgrip ${ }^{\text {ex122 }} / \mathrm{Y}$ embryos, no signal was observed (data not shown), proving the specificity of our DGrip antibody in embryo stainings.

We first looked at stage-16 embryos, in which muscles are fully attached. Here, DGrip protein is localized to both anterior and posterior edge of the VLMs, where the muscles are in contact with their attachment sites (Fig. 6A, inset, and Fig. 6B, arrowhead; for a costaining with muscle myosin, see Fig. 6F). Weaker expression of DGrip is also detected in the contact regions of more dorsal muscles attaching to the segment border (Fig. 6A, arrows), whereas no DGrip expression could be detected at the contact sites of LTMs and other directly attaching muscles. This agrees with the mRNA distribution of dgrip, which also shows strong staining in the VLM region (Fig. 1E,F, arrowheads). A GFP-tagged variant of DGrip expressed using 24B-gal4 rescued the dgrip phenotype (data not shown) and was found to accumulate at muscle edges of VLMs as well (Fig. 6G, arrowhead). We also analyzed the temporal profile of DGrip expression in embryonic muscles. Consistent with the distribution of the dgrip transcript, DGrip protein is absent from early embryos and is first detected in developing mesoderm from stage 13 on (data not shown). Obviously already in early stage 14, DGrip starts accumulating at both anterior and posterior muscle end (Fig. 6C). The protein then progressively concentrates (Fig. 6C-E) to become very sharply localized there in stage 16 (Fig. 6A,B,E). To examine DGrip expression early in an identified VLM, we again used 5053-gal4 to stain muscle 12 (muscle myosin is not yet expressed in these early stages). Even before proper attachment of muscle 12 at the segment border is established, staining at both the posterior and anterior end of muscle 12 is observed (Fig. 6H,J,L, see arrowheads in J). As expected, DGrip is sharply localized in stage 16 (Fig. 6I,K,M, see arrowheads in K,M). It should be noted that DGrip staining is certainly not restricted to the labeled VLM.

DGrip staining in embryonic muscle appears as discrete punctae, suggesting that the protein accumulates in distinct intracellular compartments (Fig. 6D,E, magnifications). To learn about its subcellular distribution, we expressed DGrip in COS-7 cells. Here, DGrip was also found expressed in discrete punctae (Fig. 6N-P), which in terms of size and distribution appeared very similar to DGrip punctae of embryonic muscles. Colocalization experiments using established markers for intracellular compartments showed a substantial overlap with markers labeling the endocytic compartment (Fig. $6 \mathrm{~N})$. In contrast, no overlap with markers of endoplasmic reticulum (Fig. 6O), Golgi (Fig. 6P), cell membrane, or other organelles as lysosomes or mitochondria (data not shown) was observed.

The presented data show that DGrip is expressed in those muscles, which are affected by the absence of the gene product. The site of DGrip localization is in agreement with the argument that the protein participates in the process of muscle guidance, possibly executing its function in an endosomal compartment. Consistent with DGrip having a transient function needed for embryonic muscle patterning, DGrip expression at muscle ends vanishes in postembryonic development (data not shown).

Overexpression of DGrip: ectopic cellular extensions on directly attaching muscles

Our study so far shows that DGrip is essential to mediate a motility response in the VLMs toward the anterior segment border. This is based on the finding that in the absence of the DGrip, the cellular extensions of developing VLMs no longer form properly. In contrast, the motility of LTMs is undisturbed in the absence of DGrip, consistent with the observation that these muscles do not seem to normally express DGrip. We thus asked whether an ectopic activity of DGrip would influence muscle motility. To achieve dgrip expression in all muscle cells, we expressed the gene in response to either 24B-gal4 or twist-gal4, both driving expression in all myogenic cells of Drosophila embryos, together with two copies of UAS-dgrip. DGrip overexpression in muscles was confirmed by immunofluorescence stainings using the anti-DGrip antibody. In embryos overexpressing the dgrip gene by using either 24B-gal4 (Fig. 7A-E) or twist-gal4 (data not shown), muscle morphology was only slightly affected in VLMs (Fig. 7B, arrowhead) and other indirectly attaching muscles. Similarly, direct overexpression of DGrip in VLM 12 using 5053gal4 was without phenotypic consequence (data not 


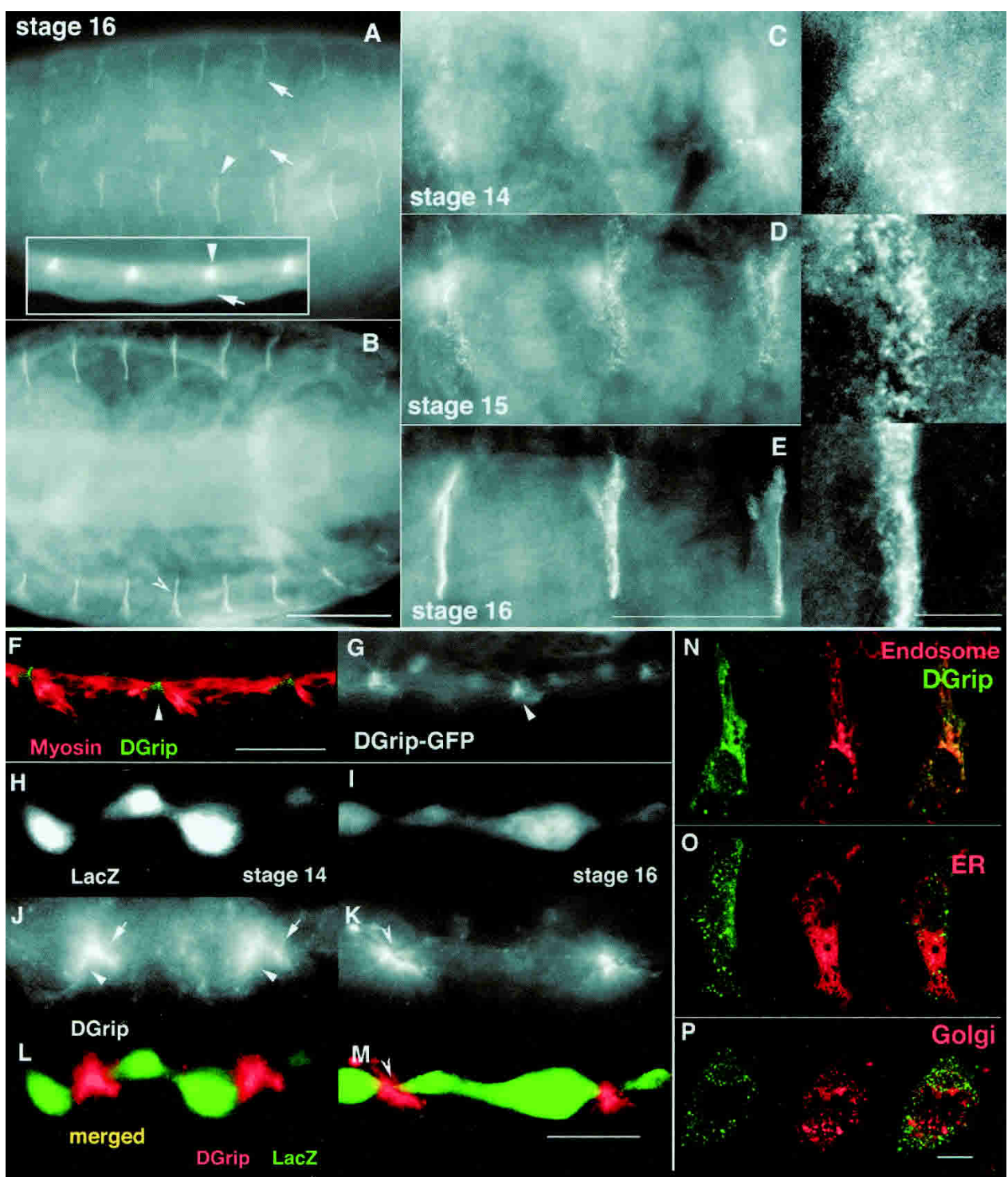

Figure 6. Expression of DGrip throughout muscle development. $(A-M)$ Immunostainings of Drosophila embryos using an affinitypurified antibody against PDZ domains 6 and 7 of DGrip (see Materials and Methods). $(A, B)$ Stage 16 wild-type embryos in lateral (A) and ventral $(B)$ perspective. DGrip is strongly expressed at contact sites of ventral longitudinal muscles (VLMs; $A, B$, arrowheads) and is weaker at segmental attachments sites of dorsal muscles attaching at the segment border ( $A$, arrows). DGrip is not observed in muscles making contacts away from the segment border. $(A$, inset) Vertical perspective on VLMs shows that DGrip expresses specifically in both anterior and posterior end of VLM muscles (arrowhead) but not in the epidermis $($ arrow). $(B) \mathrm{Bar}, 80 \mu \mathrm{m}$. $(C-E)$ Higher magnification of DGrip expression in the VLM region of stage $14(C), 15(D)$, and $16(E)$. (Insets) Further magnification. DGrip progressively accumulates at the contact sites of the muscles. (E) Bar, $30 \mu \mathrm{m}$; (inset) $3 \mu \mathrm{m}$. (F) Costaining of DGrip with muscle myosin in stage 16. DGrip expression is confined to segment border attachment sites (arrowhead); bar, $20 \mu \mathrm{m}$. (G) GFP-tagged DGrip expressed in mesoderm using 24B-gal4 is enriched at both anterior and posterior ends of muscle (arrowhead). (H-M) Costaining between endogenous DGrip $(J, K)$ and $\beta$-Gal $(H, I)$ specifically expressed in muscle 12 using 5053-gal4 together with UAS-lacZ. In stage 14, DGrip concentrates in both anterior $(H, J, L$, arrowheads in $J)$ and posterior ends $(H, J, L$, arrows in $J)$ of extending muscle 12 , which has not yet established contact with the anterior segment border. In stage $16(I, K, M)$, muscle 12 is firmly attached and DGrip expression is strongly concentrated at the segment border. $(M)$ Bar, $30 \mu \mathrm{m}$. $(N-P)$ COS 7 cell cotransfected with DGrip and compartment markers. DGrip localizes to intracellular punctae, which in size and distribution are similar to the DGrip punctae observed in embryonic muscles $(D$, inset). DGrip in COS cells overlaps with endosomal markers $(N)$. No colocalization to ER $(O)$ or Golgi $(P)$ was observed.

shown). In contrast, LTMs (Fig.7A,C-E) were very sensitive with respect to DGrip overexpression. Such LTMs adopted an irregular morphology (Fig. 7A; for controls, see Fig. 3A), bent to attach at the segment border (Fig.
7A, arrowheads), or produced thin cellular extensions, which connected them to the segment borders (Fig. 7C,D). In controls, such cellular extensions formed by LTMs or bending of whole LTMs toward segment bor- 

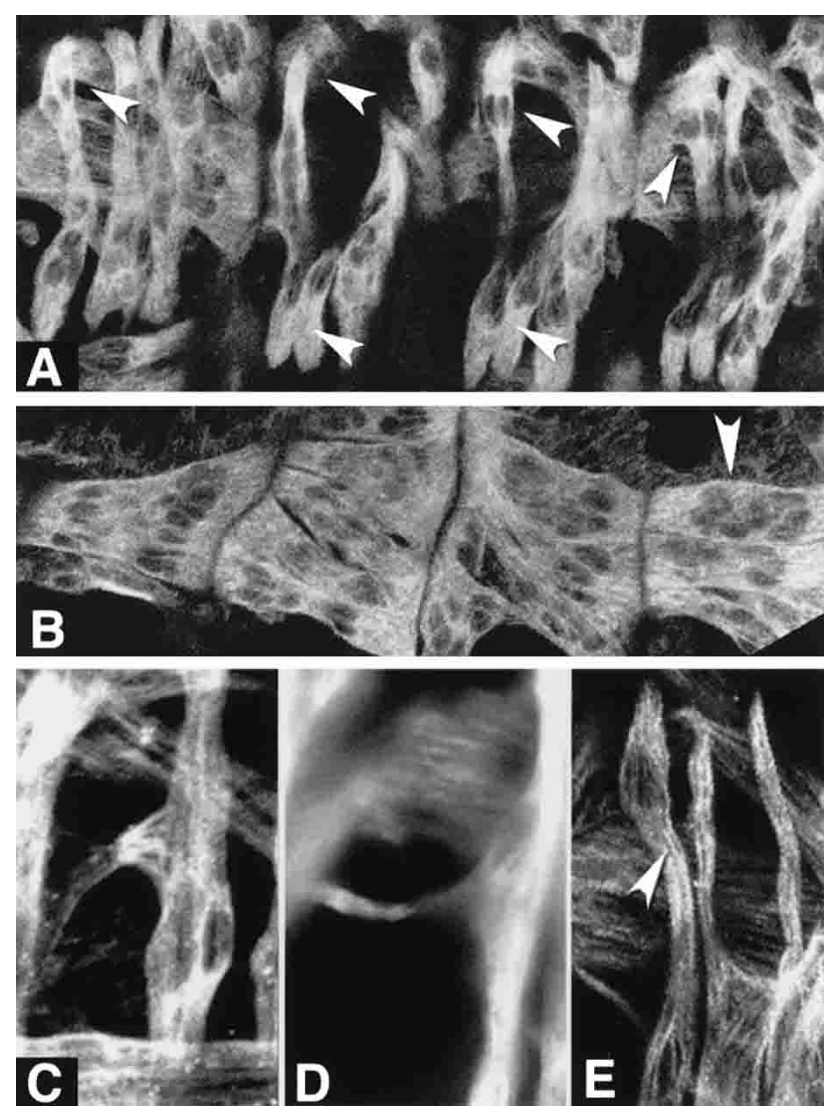

Figure 7. DGrip overexpression provokes segment border attachment of lateral transverse muscles (LTMs). All images show anti-muscle myosin stainings at stage 17 of DGrip overexpressing embryos (24B-gal4::2xUAS-dgrip). $(A, B)$ Confocal projections of hemisegments A1 to A4 from one individual embryo showing the LTM $(A)$ or VLM $(B)$ region. Although LTMs are normally oriented upright in the segments and appear bar-like (for a control embryo, see Fig. 3A), after DGrip overexpression LTMs often fully bend and attach at segment borders $(A$, arrowheads). Alternatively, these muscles form thin projections $(C-E$, arrowheads), which attach to the segment borders. In VLMs overexpressing DGrip, neither ectopic projections nor defective choice of attachment sites were observed $(B)$. (A) Bar, $30 \mu \mathrm{m}$.

ders were never observed, neither was formation of ectopic cellular extensions from directly attaching muscles overexpressing DGrip. Thus, ectopic expression of DGrip can efficiently provoke LTMs to attach to other muscles at the segment border. Muscles that display both direct and indirect attachment modes, such as the ventral oblique and acute muscles, display misrouted processes at the directly attaching end of the muscle, whereas the indirectly attaching ends of these muscles are essentially unaffected as for VLMs. Conversely, in the dgrip mutant we specifically find the indirectly attaching ends of these muscles to be affected (data not shown). No sign of ectopic tendon cell differentiation was observed in embryos overexpressing dgrip in developing muscles, indicating that misattached muscles were misguided toward preexisting segment border tendon cells (data not shown).
We conclude that DGrip is a key player in organizing different patterns of muscle attachment between different groups of muscles. The factor is both necessary and sufficient to promote the formation of muscle cell extensions, which we implicate in sensing and reacting toward a guidance signal expressed at the segment border.

\section{Discussion}

In this study we provide evidence that the only member of the GRIP family in Drosophila (DGrip) organizes cellular motility in order to allow proper attachment site guidance in embryogenesis. This is intriguing, as proteins of the GRIP family so far have mainly been described in the context of synapse formation and plasticity, and are considered to be involved in the clustering and/or transporting of synaptic proteins, most importantly glutamate receptors.

\section{Drosophila Grip is both necessary and sufficient to direct developing muscles toward segment borders}

The DGrip transcript and protein are strongly expressed within a specific subset of embryonic muscles of Drosophila, the VLMs. VLM founder cells are born in the posterior part of each segment, and they extend growthcone-like structures in the anterior direction to target to specific attachment sites corresponding to the segment borders. At these sites, the VLMs form stable connections in a tripartite complex with apodemes and other VLMs (Fig.5; Bate 1990; Bunch et al. 1998; Prokop et al. 1998; Martin-Bermudo and Brown 2000). In the absence of DGrip activity, cellular extensions of the VLMs are abnormal "from the beginning." Consequently, VLMs fail to attach at the segment borders, but instead form pure muscle-muscle contacts irrespective of their position within the segment. Often the muscles fail to recognize the segment borders. All aspects of the dgrip mutant phenotype were fully rescued in response to DGrip expression from a dgrip cDNA-containing transgene in the developing VLMs of dgrip mutant embryos. This result unambiguously establishes that the lack of dgrip activity within the growing muscle is directly responsible for the defects observed.

Elimination of GRIP1 in mice results in embryonic lethality (Bladt et al. 2002) associated with defective dermoepidermal junctions. These results were interpreted to indicate that the architecture of this contact requires PDZ domain interactions mediated through GRIP1, in order to maintain proper cell adhesion. The contact between epidermis and specific muscles is not properly formed in Drosophila embryos mutant for dgrip. This observation on first sight might hint toward a defect in the stabilization of cell adhesion in Drosophila dgrip mutants as well. However, escaping adult Drosophila from dgrip null alleles showed no signs of adhesion loss (Fig. 4J). Moreover, defects in dgrip mutants are limited to one muscle group in a way that argues against cell 
adhesion defects and favors a role of dgrip in muscle guidance, in which the pathways identified to date are found to act in a muscle subgroup-specific manner (Callahan et al. 1996; Kramer et al. 2001). Consistently, the mechanical attachment of muscles in dgrip mutants is unaffected, because the mutant VLMs form integrin-expressing attachment sites. Furthermore, the attachment was stable upon contraction, as the dgrip mutant larvae were able to locomote robustly.

Our findings exclude the possibility that the phenotypes of dgrip mutants are due to an effect on cell adhesion properties in the process of stabilizing the muscle attachment sites versus upcoming muscular contraction force. Instead, we provide direct evidence that the motility of VLMs is specifically affected in the absence of DGrip, by visualizing the morphological development of VLMs during guidance (Fig.5). Wild-type VLMs form growth-cone-like extensions invariantly projecting in the anterior direction (Fig. 5A-C). However, in dgrip mutant muscles, the direction of cellular extensions appears randomized from the beginning, and often extensions appear collapsed (Fig. 5D-F). Furthermore, upon overexpression of DGrip, ectopic cellular extensions form specifically from LTMs, which normally do not express the protein. These aberrant extensions frequently contacted and anchored at the segment borders (Fig. 7), where obviously they became stabilized as still they are detected in late larval muscles (data not shown).

\section{Does DGrip organize a novel signaling pathway controlling muscle motility?}

Our data imply that DGrip mediates a motility response within developing muscles toward an attractive signal expressed at the segment border. It has been reported that Robo receptors are required to extend toward Slitexpressing muscle attachment sites at segment borders (Kramer et al. 2001). Loss of Robo-Slit function eliminates segment border attachment in VLMs, whereas overexpression of Robos leads to segment border attachment in LTMs. Moreover, we see that Robo receptors are expressed at the edges of developing muscles in a spatiotemporal pattern very similar to the expression profile of DGrip (data not shown). Because of these obvious parallels between Robo/Slit and DGrip, we extensively addressed a potential interaction of these factors by genetic and biochemical means. No evidence for a functional or physical interaction could be obtained. It therefore appears most likely that DGrip organizes the response to a novel signal working in parallel to the Robo/Slit-system. The finding that DGrip overexpression provokes changes in LTMs, whereas Robos are reported to be absent from these muscles (Kramer et al. 2001), also argues in this direction. In principle, DGrip could be involved in the execution of a signaling event, or alternatively, it might be important for the stabilization of first interactions pioneered, for example, by Robo/Slit signaling. Because dgrip mutant muscles show defective extensions early during muscle guidance and, secondly, overexpression of
DGrip directly causes the formation of cellular extensions, we favor the first alternative.

VLM-type muscles by far show the strongest defects within dgrip mutants, affecting $100 \%$ of VLMs 6 and 7 . However, other indirectly attaching muscles did show defects as well. Although the defects were weaker in these cells than in VLMs, they clearly were significant in comparison to control animals. Consistently, although DGrip expression seems strongest at VLM attachment sites, the contacts of more dorsal muscles, which also attach indirectly, also express the protein. A similar situation, characterized by VLMs being most affected and expressing the most DGrip between the indirectly attaching muscles, is reported for the Robo/Slit muscle guidance pathway (Kramer et al. 2001). It might be that spatiotemporal specificities in the development of the VLMs make this particular muscle group especially dependent on robust guidance signaling between the indirectly attaching muscles. Drosophila muscle guidance has not so far been subject to saturating genetic analysis and besides few seminal studies (Volk and VijayRaghavan 1994; Frommer et al. 1996; Becker et al. 1997; Vorbrüggen and Jäckle 1997; Kramer et al. 2001), our understanding of the process is still rather poor. In several other models of cellular motility, for example, growth cone migration, distinct pathways partially working in parallel have also been identified (for review, see Huber et al. 2003).

\section{Mechanistic analysis of GRIP family proteins in Drosophila}

Even in the complete absence of myoblast fusion, muscle founder cells still form properly attached minimuscles (Rushton et al. 1995). Hereby, the initial polarization of these specific muscle precursors seemingly does not depend on tendon cells. However, the tendon cells provide essential guidance cues that direct muscle extension (Bate 1990; Frommer et al. 1996). It is essentially unknown, how cellular polarity is organized throughout the time course of guidance and subsequent muscle attachment. Most likely the polarized transport of relevant proteins toward the "active muscle ends" is important already early within muscle guidance (Yarnitzky et al. 1997). In fact, developing muscles display a polarized microtubule network with the + ends facing the attachment sites (Clark et al. 1997).

DGrip appears concentrated at ends of muscle cells (Fig. $6 \mathrm{H}, \mathrm{J}, \mathrm{L}$ ) before any proper attachment between the muscle and its prospective attachment site is established. As an intracellular adaptor molecule, DGrip might organize signaling processes, for example, by clustering transmembrane receptors, or it might act downstream of the actual signaling processes, for example, by executing transporting events that are essential for directed muscle cell motility. In fact, the correct targeting of the EGF receptor ligand Vein to the site of muscle tendon attachment has been shown to be an essential step in organizing proper muscle pattern (Strumpf and Volk 1998). Our data suggest that after supplying DGrip 
to muscles that normally do not express the protein, they start to a sense distant guidance cue, which in turn causes the formation of cellular extensions. DGrip thus might switch on dormant receptors in muscles, for example, by mediating their transport to relevant cellular locations. Interestingly, DGrip has been suggested to control the transport of transmembrane receptors and signaling molecules, such as glutamate receptors and ephrins, from intracellular compartments to the cell surface (Torres et al. 1998; Wyszynski et al. 1998; Brückner et al. 1999; Dong et al. 1999b; Braithwaite et al. 2002; Hirbec et al. 2003). In Drosophila muscles we find DGrip localizing to discrete punctae similar to punctae formed by DGrip in culture cells. Colocalization experiments in culture cells showed that DGrip punctae often colocalized with endosomal markers, whereas no colocalizations with ER, Golgi, plasma membrane, lysosomal, or mitochondrial markers were observed. We thus favor the hypothesis that DGrip mediates signaling throughout muscle motility by regulating the endosomal trafficking of receptor complexes. Specialized proteins regulating signaling by endosomal trafficking have recently emerged key players in animal development (for review, see Piddini and Vincent 2003). Regulation of membrane protein composition by GRIPs might be subtle, as different receptor populations such as AMPA/Kainate receptors have been suggested to be regulated by GRIP in opposing manners (Hirbec et al. 2003). Palmitoylation close to the N-terminal end has been described for the Grip family members GRIP1b and pABP-L, and is suggested to control their intracellular distribution (DeSouza et al. 2002; Yamazaki et al. 2001). Indeed, the absolute $\mathrm{N}$ terminus of DGrip contains a conserved cysteine residue at position 13 and is similar to the $\mathrm{N}$-terminal sequences demonstrated to mediate palmitoylation of GRIP1b and pABP-L. Our first experimental data in fact suggest posttranslational modification of DGrip with palmitate.

The highly penetrant embryonic phenotype of DGrip presented in this study should thus be especially well suited to further study mechanisms of GRIP function in the genetically well-tractable Drosophila model.

\section{Materials and methods}

\section{Genetics}

For dgrip mutagenesis, $\mathrm{P}(\mathrm{KG}) 02862$ (Roseman et al. 1995) or P(GT1)BG01736 (Kimmerly et al. 1996) were crossed to $\Delta 2-3$ Transposase for P-element mobilization. Deficiencies dgrip ${ }^{\text {ex36 }}$ and dgrip ${ }^{e x 122}$ were identified and mapped by using genomic PCR from hemizygous mutant larvae (detailed information on demand). To identify dgrip mutant embryos, the corresponding alleles were balanced over FM7-ftz-lacZ (Heitzler 1997) and negatively identified in $\beta$-Gal and Sex-lethal (Bopp et al. 1991) costainings. For larvae, we balanced over FM7-Act-GFP and sorted male, non-GFP larvae under a fluorescence binocular (MZFLIII, Leica). For visualization of muscle 12 in dgrip mutants, recombinant $d g r i p^{e x 36}, U A S-1 a c Z$ was balanced over FM7ftz-lacZ and virgins crossed to 5053-gal4 males (Ritzenthaler et al. 2000). In rescue experiments, dgrip ${ }^{\text {ex36 }}$ or dgrip $^{\text {ex122 }}$ was ei- ther recombined with twist-gal4 (Yin and Frasch 1998) or combined with 24B-gal4 (Brand and Perrimon 1993) and virgins crossed with homozygous UAS-dgrip males. A more weakly expressing UAS-dgrip line was chosen, and rescued animals were identified as above. For DGrip overexpression, a recombination of two strongly expressing UAS-dgrip lines (overexpression scored by anti-DGrip immunostainings; data not shown) was crossed to $24 B$-gal4 or twist-gal4.

\section{Molecular biology}

The following EST clones encoding dgrip have been isolated in the Berkeley Drosophila Genome Project: RE14068, RE32265, RE70628, and RE44067. All contain identical 5' and 3'sequence. RE14068 is fully sequenced (NCBI access no. AAL68270) and was used for conceptual translation of DGrip. For pUAST-dgrip, the DGrip ORF was amplified from EST RE14068 (BDGP, Research Genetics) by using primers 5'-ATACAAGATCTCAA GATGAAACTGTGGAAATCG- $3^{\prime}$ and $5^{\prime}$-AGTACTCGAGGC TCGGTAAAGAATACAGGA- $3^{\prime}$ and cloned BglII/Xhol into the pUAST trangenesis vector (Brand and Perrimon 1993). To express C-terminally GFP-tagged DGrip, we amplified using $5^{\prime}$ ATACAAGATCTCAAGATGA AACTGTGGAAATCG-3' and 5'-CATCTCGAGAGAGCGCTGCATGATCATCTCG-3' omitting the DGrip stop codon, subcloned BglII/XhoI into pEGFP-N1 (Clontech), and then cloned BglII/NotI into pUAST. All constructs were confirmed by double-strand sequencing and transgenic flies produced by using standard procedures.

\section{Antibody production, affinity purification, and immunodetection}

For immunogen purification, a fragment encoding PDZ domains 6 and 7 of DGrip was amplified by using primers $5^{\prime}$-GC GCCTCGAGCATGGGTGCTCCCACAAGCACAG-3' and 5'GACTCTAGACGGGCAAAAGCATCACTCAG-3', subcloned into pDNR-1 (Clontech) XhoI/XbaI, and then cloned XhoI/NotI into pGex4T-3 (Pharmacia). The GST-fusion was expressed in BL21 cells and purified on GSH-agarose (Pharmacia), following the instructions of the manufacturer. Purified protein was injected into rabbits (BioGenes). For affinity-purification of sera, a $\mathrm{XhoI} / \mathrm{XbaI}$ fragment from the pDNR-1-PDZ6-7 construct above was blunted at the $3^{\prime}$ end with Klenow enzyme and ligated into the $(\mathrm{His})_{6}$-tag vector pQE-32 (Qiagen), cut SalI/HindIII where the HindIII was also treated with Klenow. Recombinant protein was expressed in XL1-blue cells and purified on Talon resin (Clontech). One milligram of the (His) ${ }_{6}$-fusion was immobilized on a column and used for affinity-purification. Specificity of sera was tested by immunostaining and Western blotting on dgrip mutant embryos.

For immunoblotting, 5- to 15-h-old Drosophila embryos were dechorionated and homogenized in lysis buffer $(50 \mathrm{mM}$ HEPES, $60 \mathrm{mM} \mathrm{NaCl}, 3 \mathrm{mM} \mathrm{MgCl} 2,1 \mathrm{mM} \mathrm{CaCl}_{2}, 0.2 \%$ Triton X-100, $0.2 \%$ Nonidet P-40, $10 \%$ glycerol) with protease inhibitor "complete mini" (Roche) added. After centrifugation, supernatant ("supernatant", Fig. 2B) was harvested, and the pellet ("pellet" Fig. 2B) was extracted with a more stringent lysis buffer (see above but $2 \%$ Triton X-100, $2 \%$ Nonidet P-40). Samples were run on an $8 \%$ PAA gel, transferred to nitrocellulose membrane (BioRad), and probed with the anti-DGrip antibody $(1: 500)$ followed by ECL-detection (Amersham).

\section{Recombinant expression and immunohistochemistry in cell culture}

For baculovirus expression, the DGrip ORF was amplified as for pUAST-dgrip, cloned into pFastBacl (Invitrogen), and trans- 
formed into DH5 $\alpha \mathrm{BAC}$ cells. A positive baculovirus clone was selected with PCR and transfected into Sf.9 cells by using the BAC-to-BAC expression system (Invitrogen). For expression in mammalian cells, dgrip was cloned BglII/XhoI from pUASTdgrip and inserted into pEGFP-N1 (Clontech) to produce pdgripEGFP. COS-7 cells were grown in Dulbecco's modified Eagle's medium containing $10 \%$ fetal calf serum and $1 \%$ penicillin/ streptomycin on coverslips. For the colocalization studies, cells were transiently transfected by using Lipofectamine2000 (Invitrogen). The following plasmids were used for transfection in combination with pdgrip-EGFP: pEYFP-Actin, pEYFP-Endo, pEYFP-Mito, and pEYFP-ER (all Clontech). For antibody stainings, cells were fixed $24 \mathrm{~h}$ after transfection with $4 \%$ PFA for $5 \mathrm{~min}$, incubated in $-20^{\circ} \mathrm{C}$ methanol for $10 \mathrm{~min}$, permeabilized by treatment with high-salt PBS $(20 \mathrm{mM} \mathrm{NaPi}$ at $\mathrm{pH} 7.4 ; 500 \mathrm{mM} \mathrm{NaCl}$ ) containing $0.3 \%$ Triton X100 followed by an incubation with $10 \%$ normal goat serum, and then incubated for $1 \mathrm{~h}$ with primary antibody. Antibodies used were as follows: anti-MPR300 antibody for Golgi-labeling (Hybridoma Bank, Hopkins University, Iowa) and mouse antilamp1 antibody (Stefan Höning, Zentrum für Biochemie und Molekulare Zellbiologie, Göttingen) for lysosome-labeling. Cy3 and Cy5 (Molecular Probes) were used as secondary antibodies. Cells were monitored under a confocal laser-scan microscope LSM510 with META modul (Zeiss). Live imaging was performed in Tyrode solution $(150 \mathrm{mM} \mathrm{NaCl}, 5 \mathrm{mM} \mathrm{KCl}$, $10 \mathrm{mM}$ glucose, $10 \mathrm{mM}$ HEPES, $2 \mathrm{mM} \mathrm{CaCl}_{2}, 1 \mathrm{mM} \mathrm{MgCl}_{2}$ at $\mathrm{pH}$ 7.4).

\section{Stainings}

Immunocytochemistry on embryos and larvae was essentially performed as described (Lin and Goodman 1994). Antibodies were usually preadsorbed to 0 to $4 \mathrm{~h}$ Drosophila embryos. Primary antibodies were as follows: FMM5A muscle myosin monoclonal (Christoph Schuster, $1: 100)$, mouse anti- $\beta P S$-integrin (Nigg Brown, $1: 100$ ), rat anti-Delilah and guinea pig antiStripe (Becker et al. 1997; $1: 500$ ), monoclonal anti-Sex Lethal (DSHB, $1: 500)$, rabbit anti- $\beta$-Gal (Cappel, $1: 500)$, and monoclonal anti- $\beta$-Gal (Promega, $1: 500)$. Secondary antibodies were Cy3- and FITC- conjugated goat anti-mouse and goat anti-rabbit FAB (Dianova), used at $1: 200$.

Phalloidin-TRITC (Sigma) was used to visualize F-actin. Previously immunostained or freshly fixed larvae were incubated for $30 \mathrm{~min}$ in PBS/0.5\% Triton-TX-100 and 5\% normal goat serum, protected from the light. Larvae were then rinsed and mounted as normal. Confocal images were taken on a Leica TCS NT system with a 63 -fold objective (1.3 NA), 4x frame averaging, and a $\Delta \mathrm{Z}$ of $600 \mathrm{~nm}$, and image stacks were projected in maximal intensity mode.

\section{Acknowledgments}

Many thanks to Herbert Jäckle and Alf Herzig for insightful comments and critical reading of the manuscript and to Christine Quentin for excellent technical assistance. Thanks also go to Talila Volk, Nick Brown, Volker Hauke, Stefan Höning, Annette Holz, Renate Renkawitz-Pohl, Barry Dickson, and Frank Schnorrer for their generous gifts of fly stocks and reagents. S.J.S. is supported by DFG grant SI $849 / 1$.

The publication costs of this article were defrayed in part by payment of page charges. This article must therefore be hereby marked "advertisement" in accordance with 18 USC section 1734 solely to indicate this fact.

\section{References}

Adams, M.D., Celniker, S.E., Holt, R.A., Evans, C.A., Gocayne, J.D., Amanatides, P.G., Scherer, S.E., Li, P.W., Hoskins, R.A., Galle, R.F., et al. 2000. The genome sequence of Drosophila melanogaster. Science 287: 2185-2195.

Bate, M. 1990. The embryonic development of larval muscles in Drosophila. Development 110: 791-804.

Bate, M. and Rushton, E. 1993. Myogenesis and muscle patterning in Drosophila. C. R. Acad. Sci. III 316: 1047-1061.

Baylies, M.K., Bate, M., and Ruiz Gomez, M. 1998. Myogenesis: A view from Drosophila. Cell 93: 921-927.

Becker, S., Pasca, G., Strumpf, D., Min, L., and Volk, T. 1997. Reciprocal signaling between Drosophila epidermal muscle attachment cells and their corresponding muscles. Development 124: 2615-2622.

Bilder, D. 2001. PDZ proteins and polarity: Functions from the fly. Trends Genet. 17: 511-519.

Bladt, F., Tafuri, A., Gelkop, S., Langille, L., and Pawson, T. 2002. Epidermolysis bullosa and embryonic lethality in mice lacking the multi-PDZ domain protein GRIP1. Proc. Nat1. Acad. Sci. 99: 6816-6821.

Bökel, C. and Brown, N.H. 2002. Integrins in development: Moving on, responding to, and sticking to the extracellular matrix. Dev. Cell 3: 311-321.

Bopp, D., Bell, L.R., Cline, T.W., and Schedl, P. 1991. Developmental distribution of female-specific sex lethal proteins in Drosophila melanogaster. Genes \& Dev. 5: 403-415.

Braithwaite, S.P., Xia, H., and Malenka, R.C. 2002. Differential roles for NSF and GRIP/ABP in AMPA receptor cycling. Proc. Natl. Acad. Sci. 99: 7096-7101.

Brand, A.H. and Perrimon, N. 1993. Targeted gene expression as a means of altering cell fates and generating dominant phenotypes. Development 118: 401-415.

Brown, N.H. 1994. Null mutations in the $\alpha$ PS2 and $\beta$ PS integrin subunit genes have distinct phenotypes. Development 120: $1221-1231$.

- 2000. Cell-cell adhesion via the ECM: Integrin genetics in fly and worm. Matrix Biol. 19: 191-201.

Brückner, K., Pablo Labrador, J., Scheiffele, P., Herb, A., Seeburg, P.H., and Klein, R. 1999. EphrinB ligands recruit GRIP family PDZ adaptor proteins into raft membrane microdomains. Neuron 22: 511-524.

Bunch, T., Graner, M., Fessler, L., Fessler, J., Schneider, K., Kerschen, A., Choy, L., Burgess, B., and Brower, D. 1998. The PS2 integrin ligand tiggrin is required for proper muscle function in Drosophila. Development 125: 16791689.

Callahan, C.A., Bonkovsky, J.L., Scully, A.L., and Thomas, J.B. 1996. derailed is required for muscle attachment site selection in Drosophila. Development 122: 2761-2767.

Clark, I.E., Jan, L.Y., and Jan, Y.N. 1997. Reciprocal localization of Nod and kinesin fusion proteins indicates microtubule polarity in the Drosophila oocyte, epithelium, neuron and muscle. Development 124: 461-470.

Contractor, A., Rogers, C., Maron, C., Henkemeyer, M., Swanson, G.T., and Heinemann, S. 2002. Trans-synaptic Eph receptor-ephrin signalling in hippocampal mossy-fibre LTP. Science 296: 1864-1869.

DeSouza, S., Fu, J., States, B.A., and Ziff, E.B. 2002. Differential palmitoylation directs the AMPA receptor-binding protein $\mathrm{ABP}$ to spines or to intracellular clusters. J. Neurosci. 22: 3493-3503.

Dickson, B.J. 2002. Molecular mechanisms of axon guidance. Science 298: 1959-1964.

Dong, H., O’Brien, R.J., Fung, E.T., Lanahan, A.A., Worley, P.F., 
and Huganir, R.L. 1997. GRIP: A synaptic PDZ domain-containing protein that interacts with AMPA receptors. Nature 386: $279-284$.

Dong, H., Zhang, P., Liao, D., and Huganir, R.L. 1999a. Characterization, expression, and distribution of GRIP protein. Ann. N. Y. Acad. Sci. 868: 535-540.

Dong, H., Zhang, P., Song, I., Petralia, R.S., Liao, D., and Huganir, R.L. 1999b. Characterization of the glutamate receptor-interacting proteins GRIP1 and GRIP2. I. Neurosci. 19: 6930-6941.

Frommer, G., Vorbrüggen, G., Pasca, G., Jackle, H., and Volk, T. 1996. Epidermal egr-like zinc finger protein of Drosophila participates in myotube guidance. EMBO J. 15: 1642-1649.

Heitzler, P. 1997. New FM7 versions from Strasbourg. DIS 80: 103 .

Hirbec, H., Francis, J.C., Lauri, S.E., Braithwaite, S.P., Coussen, F., Mulle, C., Dev, K.K., Couthino, V., Meyer, G., Isaac, J.T.R., et al. 2003. Rapid and Differential regulation of AMPA and Kainate receptors at hippocampal mossy fibre synapses by PICK1 and GRIP. Neuron 37: 625-638.

Huber, A.B., Kolodkin, A.L., Ginty, D.D., and Cloutier, J.F. 2003. Signaling at the growth cone: Ligand-receptor complexes and the control of axon growth and guidance. Ann. Rev. Neurosci. 26: 509-563.

Jagla, K., Bellard, M., and Frasch, M. 2001. A cluster of Drosophila homeobox genes involved in mesoderm differentiation programs. Bioessays 23: 125-133.

Jesaitis, L.A. and Goodenough, D.A. 1994. Molecular characterization and tissue distribution of $\mathrm{ZO}-2$, a tight junction protein homologous to ZO-1 and the Drosophila discs-large tumor suppressor protein. J. Cell Biol. 24: 949-961

Kimmerly, W., Stultz, K., Lewis, S., Lewis, K., Lustre, V., Romero, R., Benke, J., Sun, D., Shirley, G., Martin, C., et al. 1996. A P1-based physical map of the Drosophila euchromatic genome. Genome Res. 6: 414-430.

Knirr, S., Azpiazu, N., and Frasch, M. 1999. The role of the NK-homeobox gene slouch (S59) in somatic muscle patterning. Development 126: 4525-4535.

Ko, J., Kim, S., Valtschanoff, J.G., Shin, H., Lee, J.-R., Sheng, M., Premont, R.T., Weinberg, R.J., and Kim, E. 2003. Interaction between Liprin-a and GIT1 is required for AMPA receptor targeting. J. Neurosci. 23: 1667-1677.

Kramer, S.G., Kidd, T., Simpson, J.H., and Goodman, C.S. 2001. Switching repulsion to attraction: Changing responses to slit during transition in mesoderm migration. Science 292: $737-$ 740.

Leptin, M., Bogaert, T., Lehmenn, R., and Wilcox, M. 1989. The function of PS integrins during Drosophila embryogenesis. Cell 56: 401-408.

Lin, D.M. and Goodman, C.S. 1994. Ectopic and increased expression of Fasciclin II alters motoneuron growth cone guidance. Neuron 13: 507-523.

Lin, D., Gish, G.D., Songyang, Z., and Pawson, T. 1999. The carboxyl terminus of B class ephrins constitutes a PDZ domain binding motif. J. Biol. Chem. 274: 3726-3733.

Littleton, J.T. and Ganetzky, B. 2000. Ion channels and synaptic organization: Analysis of the Drosophila genome. Neuron 26: $35-43$.

MacKrell, A.J., Blumberg, B., Haynes, S.R., and Fessler, J.H. 1988. The lethal myospheroid gene of Drosophila encodes a membrane protein homologous to vertebrate integrin $\beta$ subunits. Proc. Nat1. Acad. Sci. 85: 2633-2637.

Martin-Bermudo, M.D. and Brown, N.H. 2000. The localized assembly of extracellular matrix integrin ligands requires cell-cell contact. J. Cell Sci. 113: 3715-3723.
Osten, P., Khatri, L., Perez, J.L., Köhr, G., Giese, G., Daly, C., Shulz, T.W., Wensky, A., Lee, L.M., and Ziff, E.B. 2000. Mutagenesis reveals a role for ABP/GRIP binding to GluR2 in synaptic surface accumulation of the AMPA receptor. Neuron 27: 315-325.

Piddini, E. and Vincent, J.P. 2003. Modulation of developmental signals by endocytosis: Different means and many ends. Curr. Opin. Cell. Biol. 15: 474-481

Prokop, A., Martin-Bermudo, M.D., Bate, M., and Brown, N.H. 1998. Absence of PS integrins or laminin A affects extracellular adhesion, but not intracellular assembly, of hemiadherens and neuromuscular junctions in Drosophila embryos. Dev. Biol. 196: 58-76.

Ritzenthaler, S., Suzuki, E., and Chiba, A. 2000. Postsynaptic filopodia in muscle cells interact with innervating motoneuron axons. Nat. Neurosci. 3: 1012-1017.

Roseman, R.R., Johnson, E.A., Rodesch, C.K., Bjerke, M., Nagoshi, R.N., and Geyer, P.K. 1995. A P element containing suppressor of hairy-wing binding regions has novel properties for mutagenesis in Drosophila melanogaster. Genetics 141: 1061-1074.

Rubin, G.M., Hong, L., Brokstein, P., Evans-Holm, M., Frise, E., Stapleton, M., and Harvey, D.A. 2000. A Drosophila complementary DNA resource. Science 287: 2222-2224.

Rushton, E., Drysdale, R., Abmayr, S.M., Michelson, A.M., and Bate, M. 1995. Mutations in a novel gene, myoblast city, provide evidence in support of the founder cell hypothesis of Drosophia muscle development. Development 121: 1979_ 1988.

Sheng, M. and Sala, C. 2001. PDZ domains and the organization of supramolecular complexes. Ann. Rev. Neurosci. 24: 1-29.

Shi, S., Hayashi, Y., Esteban, J.A., and Malinow, R. 2001. Subunit-specific rules governing AMPA receptor trafficking to synapses in hippocampal pyramidal neurons. Cell 105: 331343.

Srivastava, S., Osten, P., Vilim, F.S., Khatri, L., Inman, G., States, B., Daly, C., DeSouza, S., Abagyan, R., Valtschanoff, J.G., et al. 1998. Novel anchorage of GluR2/3 to the postsynaptic density by the AMPA receptor-binding protein ABP. Neuron 21: 581-591.

Strumpf, D. and Volk, T. 1998. Kakapo, a novel cytoskeletalassociated protein is essential for the restricted localization of the neuregulin-like factor, vein, at the muscle-tendon junction site. J. Cell Biol. 143: 1259-1270.

Torres, R., Firestein, B.L., Dong, H., Staudinger, J., Olson, E.N., Huganir, R.L., Bredt, D.S., Gale, N.W., and Yancopoulos, G.D. 1998. PDZ proteins bind, cluster, and synaptically colocalize with Eph receptors and their ligands. Neuron 21: $1453-1463$.

Volk, T. 1999. Singling out Drosophila tendon cells: A dialogue between two distinct cell types. Trends Genet. 15: 448453.

Volk, T. and VijayRaghavan, K. 1994. A central role for epidermal segment border cells in the induction of muscle patterning in the Drosophila embryo. Development 120: 59-70.

Vorbrüggen, G. and Jäckle, H. 1997. Epidermal muscle attachment site-specific target gene expression and interference with myotube guidance in response to ectopic stripe expression in the developing Drosophila epidermis. Proc. Natl. Acad. Sci. 94: 8606-8611.

Willott, E., Balda, M.S., Fanning, A.S., Jameson, B., Van Itallie, C., and Anderson, J.M. 1993. The tight junction protein ZO-1 is homologous to the Drosophila discs-large tumor suppressor protein of septate junctions. Proc. Natl. Acad. Sci. 90: 7834-7838. 
Wyszynski, M., Kim, E., Yang, F.C., and Sheng, M. 1998. Biochemical and immunocytochemical characterization of GRIP, a putative AMPA receptor anchoring protein, in rat brain. Neuropharmacology 37: 1335-1344.

Wyszynski, M., Valtschanoff, J.G., Naisbitt, S., Dunah, A.W., Kim, E., Standaert, D.G., Weinberg, R., and Sheng, M. 1999. Association of AMPA receptors with a subset of glutamate receptor- interacting protein in vivo. J. Neurosci. 19: 65286537.

Wyszynski, M., Kim, E., Dunah, A.W., Passafaro, M., Valtschanoff, J.G., Serra-Pages, C., Streuli, M., Weinberg, R.J., and Sheng, M. 2002. Interaction between GRIP and Liprina/SYD2 is required for AMPA receptor targeting. Neuron 34: $39-52$.

Xia, J., Chung, H.J., Wihler, C., Huganir, R.L., and Linden, D.J. 2000. Cerebellar long-term depression requires PKC-regulated interactions between GluR2/3 and PDZ domain-containing proteins. Neuron 28: 499-510.

Yamazaki, M., Fukaya, M., Abe, M., Ikeno, K., Kakizaki, T., Watanabe, M., and Sakimura, K. 2001. Differential palmitoylation of two mouse glutamate receptor interacting protein 1 forms with different N-terminal sequences. Neurosci. Lett. 304: 81-84.

Yarnitzky, T., Min, L., and Volk, T. 1997. The Drosophila neuregulin homolog Vein mediates inductive interactions between myotubes and their epidermal attachment cells. Genes \& Dev. 11: 2691-2700.

Yin, Z. and Frasch, M. 1998. Regulation and function of tinman during dorsal mesoderm induction and heart specification in Drosophila. Dev. Genet. 22: 187-200. 


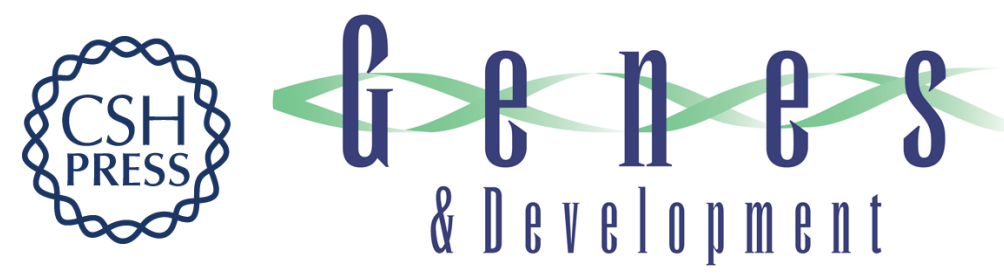

\section{A Glutamate Receptor-Interacting Protein homolog organizes muscle guidance in Drosophila}

Laura E. Swan, Carolin Wichmann, Ulrike Prange, et al.

Genes Dev. 2004, 18:

Access the most recent version at doi:10.1101/gad.287604

$\begin{array}{ll}\text { References } & \text { This article cites } 62 \text { articles, } 31 \text { of which can be accessed free at: } \\ \text { http://genesdev.cshlp.org/content/18/2/223.full.html\#ref-list-1 }\end{array}$

License

Email Alerting Receive free email alerts when new articles cite this article - sign up in the box at the top Service right corner of the article or click here.

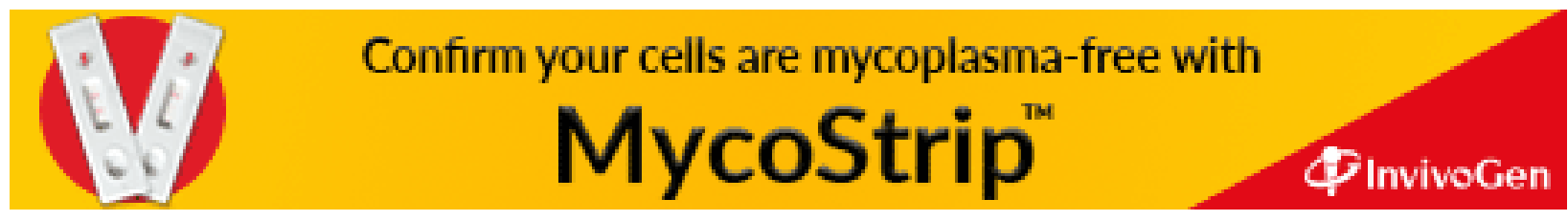

